\title{
Confining Liquids on Silicon Surfaces to Lubricate MEMS
}

\author{
Jonathan Y. Leong ${ }^{1} \cdot$ Jie Zhang $^{2} \cdot$ Sujeet K. Sinha $^{3} \cdot$ Andrew Holmes $^{4}$. \\ Hugh Spikes $^{2} \cdot$ Tom Reddyhoff ${ }^{2}$
}

Received: 14 November 2014/ Accepted: 8 April 2015/Published online: 23 May 2015

(c) The Author(s) 2015. This article is published with open access at Springerlink.com

\begin{abstract}
Liquid lubrication may provide a solution to the problem of high friction and wear in micro-electromechanical systems. Although the effectiveness of this approach has been demonstrated in laboratory-based friction tests, practical constraints prevent it from being applied in commercial devices. The main problem is how to position the lubricant on a silicon surface in order to limit spreading and evaporation. This paper describes two techniques to address this issue. First, low concentrations of additives are used to promote autophobic behaviour. Tests' results show that certain concentrations of both multiply alkylated cyclopentane and amine additives are effective in halting the spread of hexadecane on silicon, and, in the latter case, cause the hexadecane drop to subsequently retract. The second approach involves applying a micro-contact printing technique previously used on gold surfaces. Here, silicon surfaces are coated with octadecyltrichlorosilane mono-layers that are then selectively removed, using oxygen plasma, to leave regions of contrasting surface energy. Results from spin tests show that surfaces treated in this way can anchor $1 \mu$ drops of
\end{abstract}

Tom Reddyhoff

t.reddyhoff@imperial.ac.uk

1 SIM University, 461 Clementi Road, Singapore 599491, Singapore

2 Tribology Group, Department of Mechanical Engineering, Imperial College London, London SW7 2AZ, UK

3 Department of Mechanical Engineering, Indian Institute of Technology Delhi, Hauz Khas, New Delhi 110016, India

4 Optical and Semiconductor Devices Group, Department of Electrical and Electronic Engineering, Imperial College London, London SW7 2AZ, UK hexadecane and water when forces of up to 22 and $230 \mu \mathrm{N}$, respectively, are applied.

Keywords Contact angle $\cdot$ Hexadecane $\cdot$ MEMS . Silicon $\cdot$ Spreading $\cdot$ Wetting

\section{Introduction}

\subsection{MEMS Lubrication}

Micro-electro-mechanical systems (MEMS) are sub-millimetre-sized machines, which have come about as a result of advances in semiconductor fabrication. Typical MEMS devices include airbag accelerometers, gyroscopes in smartphones and implanted drug delivery metres. The MEMS industry is currently worth around 10 billion dollars and is predicted to exceed 20 billion in 2017 [1]. Furthermore, their low cost, high tolerances and ability to combine sensors and actuators with microprocessors, give MEMS the potential to profoundly affect our way of life.

Unfortunately, significant levels of friction and wear occur in MEMS, due to their high surface-to-volume ratio and the fact that silicon-the most common material in MEMS - is brittle and has high surface energy. As a result of these problems, current commercial MEMS designs are confined to non-, or very low-sliding devices [2]. This precludes the possibility of rotating or reciprocating MEMS such as micro-engines and micro-generators.

Research efforts to tackle this problem have suggested various lubrication methods. Self-assembled mono-layers have been shown to reduce adhesion, but are unable to prevent failure under sliding conditions [3]. Coatings such as DLC have shown some success [4] but are also unable to resist prolonged sliding. Vapour phase lubrication, whereby 
an alcoholic vapour is used to form a self-replenishing film on the sliding surfaces [5], has also been suggested. This is effective at reducing wear but requires hermetic sealing and shows higher friction than when liquid lubricants are used [6]. A promising approach is liquid lubrication, whereby a liquid is entrained between sliding surfaces causing a pressure field to develop that acts to separate the components. This was initially ignored in MEMS due to the assumption that viscous drag forces would be too high [7]. However, it has been shown that liquid lubrication is effective at controlling friction and wear in MEMS, provided very low viscosity lubricants are used [8]. Furthermore, the fluid film (i.e. hydrodynamic) friction in MEMS can be further reduced by inducing apparent slip-like behaviour caused by multiply alkylated pentane additives [9]. At low speeds, liquid lubrication suffers from high boundary friction that results when there is insufficient entrainment of fluid to separate the sliding surfaces. However, this problem has been solved using low concentrations of amine additives that function as friction modifiers [10].

To date, research on liquid lubrication of MEMS has been carried out on a model, silicon MEMS-type contact, but has yet to be implemented in a working MEMS device. Before this is possible, a number of practical aspects need to be addressed, including how to position the lubricant close to the contact and prevent it escaping. To this end, the research described in this paper explores two methods of containing liquids in MEMS. These methods of surface modification were originally developed for other areas, such as micro-contact printing $(\mu \mathrm{Cp})$, and are adapted to function with lubricants (hexadecane and water) and silicon wafer surfaces.

Although this research is motivated by MEMS devices, it is also relevant to the lubrication of watch and instrument bearings as well as magnetic recording applications.

\subsection{Liquid Spreading and Containment}

The kinetics of liquid drops on surfaces has been studied extensively in a wide range of applications for over a century. Instead of attempting to summarise all this work, this section will focus on autophobicity and spreading prevention methods, since these may be applied to confine liquid drops on Si surfaces. For a broader perspective, see extensive reviews by Rosen [11], Fowkes [12] and Bonn et al. [13].

Research on the spreading of liquids took off in the second quarter of the twentieth century. In 1925, Woog patented a method to prevent liquid from spreading away from watch bearings that consisted of applying a boundary of stearic acid (known as an epilame) [14, 15]. Following this, Bulkley and Snyder [16] observed that fatty acids wetted metal surfaces less well than mineral oils with apparently similar properties and attributed this anomalous behaviour to the adsorption of surface films. This was further clarified by Zisman and co-workers who used small concentrations of fatty alcohols in hexadecane to modify the degree of wetting of hexadecane on glass surfaces. They deduced that the fatty alcohols adsorbed to form a low-energy monolayer on the glass surface, which prevented the hexadecane from wetting (thereby discovering self-assembled monolayers) [17]. Zisman later coined the term "autophobic" to describe such solutions [18], and to distinguish them from liquids whose high cohesion is the sole cause that prevents them from spreading [19]. This understanding was applied by Bernett and Zisman [20] to prevent the spreading of liquids on components, where they report that "Spreading of liquid over solid surfaces can be prevented by three approaches: The liquid can be inherently non-spreading in the pure state, it can be made nonspreading by addition of carefully selected solutes or the solid surface can be modified by coating it with substances of low surface energy".

These methods of controlling the spread of liquid developed by Zisman and co-workers are highly relevant to current MEMS applications; however, they must be modified to apply to silicon surfaces rather than steels. Thus, surfactants such as fatty acids must be replaced by those which are attracted to silicon surfaces.

More recently, a considerable amount of research has focussed on droplet behaviour, driven by various modern applications such as thiol drops on gold in micro-contact printing $(\mu \mathrm{Cp})$ [21], molten alloy drops used in soldering [22], water drops on glass windows [23] and protein solutions for biomedical applications [24]. The only efforts aimed at preventing liquid spreading on MEMS surfaces have been the development of the "Localised Lubrication" technique in which a syringe and needle are used to dispense a single drop of PFPE solution onto a silicon wafer surface to provide lubrication at a precise location [25, 26]. In these recent studies, the goal has typically been to model and predict droplet behaviour. In the cases where the aim has been to control droplet movement, the methods employed are based largely on the principles outlined by Zisman above.

An additional way to confine lubricant is to modify the surface geometry, either by machining a trough/moat on the component or positioning an edge close to the drop that prevents it from flowing [27]. These methods may be of practical importance to MEMS applications, since they can easily be applied during the silicon fabrication process. Although not tested in the current work, these will be the subject of future study.

\subsection{Theoretical Background}

This section presents the theory behind this research. Although the analysis is basic, it is directly applicable to 
MEMS lubrication. Furthermore, with exceptions such as the excellent texts by Mate [28] and Brochard-Wyart et al. [29], there are few places where such information is presented clearly from a tribological perspective.

The behaviour of a drop of liquid on a surface is governed by the following parameters. The surface free energy of a solid, $\gamma_{S}$, is defined as the work per unit area required to create new surface, and gives a measure of how attractive the surface is to a liquid. The equivalent property for a liquid is known as cohesion, $\gamma_{\mathrm{L}}$, and gives an indication of the liquid's propensity to reduce its surface area by forming a spherical configuration. When a drop of liquid is placed on a surface, energy is released as the two phases interact and form an interface of energy $\gamma_{\mathrm{SL}}$. Young's well-known equation shows how these three energies balance at the contact line (perimeter) of a liquid drop on a surface (i.e. the system reaches equilibrium when the surface energy released at the solid-vapour interface equals the energy expended in increasing the surface area of the liquidvapour interface):

$\gamma_{\mathrm{S}}=\gamma_{\mathrm{L}} \cos \theta+\gamma_{\mathrm{SL}}$

where $\theta$ is the contact angle, which adjusts itself depending on the balance of energies. Therefore, if $\gamma_{\mathrm{S}}$ is sufficiently high compared to $\gamma_{\mathrm{L}}$ and $\gamma_{\mathrm{SL}}, \theta$ reduces to zero (complete wetting occurs) and the drop spreads until volumetric constraints finally limit its motion. More precisely, a liquid is considered to completely wet a surface when the contact angle is close to zero. Rearranging Eq. (1) shows conditions for this to occur:

$0<\gamma_{S}-\left(\gamma_{S L}+\gamma_{L}\right)$

where the quantity on the right-hand side of the inequality is known as the spreading parameter, $S(S>0$ implies the drop will spread over the surfaces). Zisman found empirically that, for a given low-energy surface, $\gamma_{S}$, wetting actually depended on $\gamma_{\mathrm{L}}$ alone [30]. This led to the definition of the term critical surface tension, $\gamma_{\mathrm{C}}$, as a means of characterising a solid surface-i.e. for a given surface $\gamma_{\mathrm{L}}<\gamma_{\mathrm{C}}$ implies spreading.

The first part of this paper describes simple experiments in which drops of lubricant are placed on a silicon surface, while parameters in Eq. (2) (namely $\gamma_{\mathrm{SL}}$ and $\gamma_{\mathrm{S}}$ ) are adjusted by means of varying additive concentration in order to limit its spreading.

Equation 1 relates a droplet subject to no external forces (spreading is driven only by interactions between surface and interfacial energies). If, however, a drop is subject to a body force (e.g. gravity or inertia), the drop will acquire an asymmetric shape that resists flow. The relationship between the force per unit length of the contact line and geometry was derived by Wolfram and Faust [31]
$F=\gamma_{\mathrm{L}}\left(\cos \theta_{\mathrm{r}}-\cos \theta_{\mathrm{a}}\right)$

where $\theta_{\mathrm{a}}$ and $\theta_{\mathrm{r}}$ are, respectively, the advancing and receding contact angles. The difference between $\theta_{\mathrm{a}}$ and $\theta_{\mathrm{r}}$ is known as contact angle hysteresis and results from the fact that, for practical surfaces, the energy released by creating a solid/liquid interface (in front of the drop) is less than the energy expended separating the liquid from the solid at the rear of the drop. Further information on contact angle hysteresis can be found in two reviews [32, 33]. Interestingly, it has recently been suggested that contact angle hysteresis is an important parameter in determining hydrodynamic friction [34]. This makes sense since separating fluid from, and subsequently joining fluid to, a solid is effectively what happens in a bearing. Hence, the difference in energy between these two processes manifests itself in friction.

If, as shown in Fig. 1, a component's surface is modified so that its energy in the region outside the drop perimeter, $\gamma_{S}^{\mathrm{a}}$, is different from that of the surface within the drop perimeter, $\gamma_{S}^{r}$, then Eqs. (1) and (3) can be combined to show that the force per unit length of the contact line will be given by

$F=\gamma_{\mathrm{S}}^{2}-\gamma_{\mathrm{S}}^{1}+\gamma_{\mathrm{SL}}^{1}-\gamma_{\mathrm{SL}}^{2}$

where superscripts refer to the two surfaces shown in Fig. 1.

The second part of this paper is concerned with modifying the parameters in Eq. 4 (i.e. reducing $\gamma_{S}^{1}$ and $\gamma_{\mathrm{SL}}^{2}$ relative to $\gamma_{\mathrm{S}}^{2}$ and $\gamma_{\mathrm{SL}}^{1}$ ) in order to increase the force required to move the drop, hence anchoring it to the surface.

\section{Experimental Details}

This section outlines the two experimental approaches that were taken, i.e. (1) low concentrations of additives were blended with hexadecane, and their effect was assessed by performing spreading tests, and (2) surfaces were selectively modified, and the resulting ability to anchor liquid drops was assessed by performing contact angle measurements and spin tests.

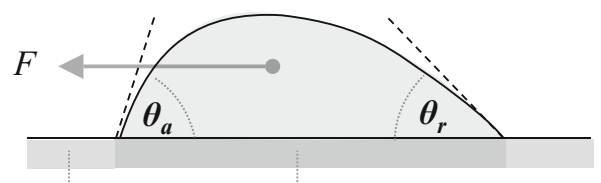

Surface $1 \quad$ Surface 2

Fig. 1 Schematic diagram of liquid drop subjected to a body force on a substrate with non-uniform surface energy 


\subsection{Materials}

Polished silicon wafers, with a $\left\langle\begin{array}{llll}1 & 0 & 0\end{array}\right\rangle$ crystal orientation and thickness $525 \mu \mathrm{m}$, were cut into squares of approximately $10 \mathrm{~mm}$ by $10 \mathrm{~mm}$ for the spin tests and $30 \mathrm{~mm}$ by $30 \mathrm{~mm}$ for the spreading tests. These were cleaned ultrasonically, first in toluene for $30 \mathrm{~min}$, followed by isopropanol for a further $30 \mathrm{~min}$ (being dried with compressed dry nitrogen gas after each immersion), before being stored in a sealed environment prior to testing.

The two liquid lubricants tested were water and hexadecane, since these have sufficiently low viscosities to give low friction in hydrodynamically lubricated MEMS contacts $[6,8-10]$.

\subsection{Liquid Modification}

Three additives were used in order to control spreading behaviour. These were octadecylamine, ODA; dodecylamine, DDA (both obtained from Sigma-Aldrich Ltd); and a multiply alkylated cyclopentane, MAC. The latter is the commercially available Nye Synthetic Oil 2001A-a mixture of di- and tri-(2-octyldodecyl)-cyclopentane (obtained from Dulub Lubricants Pte Ltd). The amine additives, ODA and DDA, were dissolved in hexadecane at concentrations of 0.1 and $0.2 \mathrm{wt} \%$, and $0.2,0.5,1.0 \mathrm{wt} \%$ respectively. Higher concentrations of DDA were obtainable compared to ODA due to the different solubility limits of the two additives. Due to the acidity of the native silicon oxide and the basic nature of amines, these amines form adsorbed surface films [10]. These concentrations were chosen since they have been shown to cause surface films to form relatively rapidly without affecting the bulk liquid properties appreciably. A range of concentrations was used in order to vary the rate of film formation and hence control spreading behaviours (higher concentrations form films more rapidly as the additive molecules require less time to diffuse through the liquid and reach the surface). The MAC lubricant is also believed to form a surface film on $\mathrm{SiO}_{2}[9$, 35 ] and was tested at a concentration of $3 \mathrm{wt} \%$, since this was found to be optimum in reducing hydrodynamic friction in previous research [9].

The purpose of using MAC and amine additives was to form a surface film and alter the degree of wetting. However, it is possible that these additives may also have the effect of altering the surface tension of hexadecane. To check whether this was the case, the surface tension of each of the additive blends was measured using a simple falling drop technique [36]. This showed that the addition of MAC or amine had a negligible effect on surface tension, and therefore, any observed effect on spreading behaviour can be attributed to solid/liquid interactions. Measured surface tension measurements of all blends showed a mean of 21.5 and standard deviation of $0.11 \mathrm{mN} / \mathrm{m}$.

\subsection{Spreading Tests}

Spreading tests were conducted by dropping $5 \mu \mathrm{l}$ portions of test lubricant onto a cleaned silicon wafer and recording their subsequent motion using a video camera. Evolution of the wetted area was extracted from the recorded video frames using a MATLAB programme, which used edge detection to count the number of pixels of lubricant and a calibrated scale to calculate the area. Tests were conducted in the laboratory with an ambient temperature of $25 \pm 2{ }^{\circ} \mathrm{C}$, at approximately $35 \%$ relative humidity.

\subsection{Surface Modification}

Self-assembled monolayers were produced using a wellestablished procedure in which $\mathrm{Si}$ wafer specimens were immersed in a solution of toluene and octadecyltrichlorosilane (OTS, $\mathrm{C}_{18} \mathrm{H}_{3} 7 \mathrm{SiCl}_{3}$, obtained from SigmaAldrich Pte Ltd) [37]. The OTS solution had a concentration of $5 \mathrm{mM}$, and the wafers were immersed for $5 \mathrm{~h}[38$, 39]. The wafers were then sonicated for $7 \mathrm{~min}$ in fresh toluene followed by ethanol to remove all non-chemisorbed OTS molecules, before being dried with compressed dry nitrogen gas.

The OTS was selectively removed using a technique similar to that developed by Lin et al. [40]. A PDMS template was prepared consisting of a flat sheet with a 2-mm-diameter circular hole. This was pressed against the wafer and then exposed to oxygen plasma for 10 min using a Harrick Plasma Cleaner at $30 \mathrm{~W}$. This exposed a circular region of the wafer to plasma, where the oleophobic OTS was removed leaving an oleophilic Si surface. The silicon wafer and PDMS mould were then separated and the former left overnight for the surface to stabilise before testing. A schematic of the three surfaces thus prepared are shown in Fig. 2.

To ascertain whether surfaces were appropriately modified prior to testing, droplet profiles and contact angles for water and hexadecane were measured using optical microscopy and Image J software with the low-bond axisymmetric drop shape analysis (LB-ADSA) plugin. These

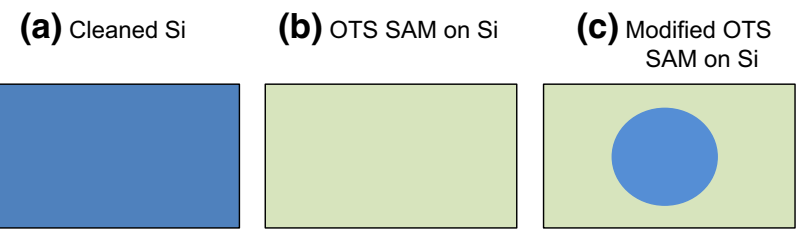

Fig. 2 Schematic diagram of modified surfaces, a after cleaning, b after OTS SAM coating, $\mathbf{c}$ after selective modification using PDMS masking and plasma treatment 
measurements were made in five locations on each sample using $5 \mu$ of liquid.

\subsection{Spin Tests}

Spin tests were conducted on a rotating plate (Fig. 3) to determine the force required to move a drop of lubricant from its original position on the wafer. In each test, the plate was spun with increasing angular speed until the drop moved from its original position. The angular velocity was increased gradually to simulate quasi-static conditions, and the sensitivity was controlled by varying the radial distance of the drop location from the axis of rotation. The centrifugal force on the drop at the instant it moved was calculated using the following formula:

$F=\frac{m \omega^{2}}{R}$

where $m$ is the mass of the droplet, $R$ the initial distance from the axis of rotation and $\omega$ the critical angular velocity (i.e. the velocity when the droplet started to move). The force obtained in this way gives a measure of how effective surface modification is at constraining the lubricant droplet.

Specimens were spun at radial distances of 20 and $40 \mathrm{~mm}$ from the centre of rotation, using drop of 1 and $2 \mu \mathrm{l}$ in volume. Each measurement was repeated five times and an average taken.

\section{Results and Discussion}

\subsection{Spreading Tests of Additive Solutions}

In the spreading tests of hexadecane blends on Si surfaces, four types of behaviours were observed, similar to those described by Cottington et al. [41] for stainless steel surfaces:

1. The drop spread with a monotonically increasing radius.

2. The drop spread initially and then retracted towards its centre, reducing in area.

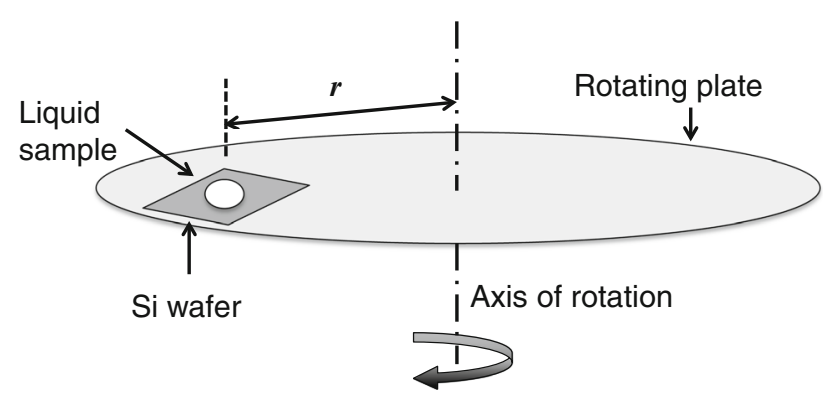

Fig. 3 Schematic diagram of experimental setup used for spin tests
3. The drop spread initially, and then violently formed one or several droplets, with a substantial contact angle, that moved rapidly away from the original location of the drop. The path of the droplet(s) avoided portions of the surface that had previously been covered.

4. The drop remained non-spreading and exhibited a substantial contact angle.

Type 1 spreading occurred only for neat hexadecane or low concentration blends. In this case, the surface tension of the liquid is less than the critical surface tension of the silicon surface, $\gamma_{C}$. This type of spreading is counterproductive in terms of lubricant containment.

Type 2 spreading, in which the droplet expands and then contracts, is exemplified in Fig. 4, where video frames from a test of $0.2 \mathrm{wt} \%$ ODA in hexadecane are shown. In this case as explained by Cottington et al. [41], once the droplet is placed on the wafer, a "foot" or meniscus is present at its base. At this initial stage, the amine layer close to the expanding perimeter (being limited by mass transport of additive to surface) does not form densely or rapidly enough to reduce the critical surface tension of the surface below that of the solution. Therefore, the drop spreads as pure hexadecane would. However, with time, additional additive molecules from solution complete the monolayer beneath the droplet so that $\gamma_{C}<\gamma_{L}$. This effect is coupled with the reduction in speed of the advancing liquid/solid/vapour boundary (that occurs due to the diffusion process that drives spreading) so that the boundary eventually halts and then retracts. The retraction of the drop usually initiates at a single location, as indicated by the arrow in Fig. 4a (possibly as a result of a surface imperfection).

Type 3 spreading, (also known as "reactive autophobic spreading" [22, 42], or "catastrophic spreading" [43]) is exemplified in Fig. 5. Such behaviour was explained by Biebuyck and Whitesides (for thiol solutions on gold surfaces) [21] and is similar in mechanism to type 2 spreading. However, a difference in contact angle between the advancing edge of the drop (where the liquid wets the surface) and the receding edge (where an ordered oleophobic monolayer has had time to form) gives rise to a negative contact angle hysteresis. As shown by Eq. 3, this results in a force which propels the drop across the surface. It was suggested that a difference in surface tension may also play a role in this type of spreading (i.e. by the Marangoni effect) [41, 43]; however, as outlined above, the additives used in this study have negligible effect on surface tension. Although remarkable, this reactive spreading is highly impractical in terms of lubricant containment and was not further pursued in this study. It should also be noted that the dark band, observed around the edge of the drops in 

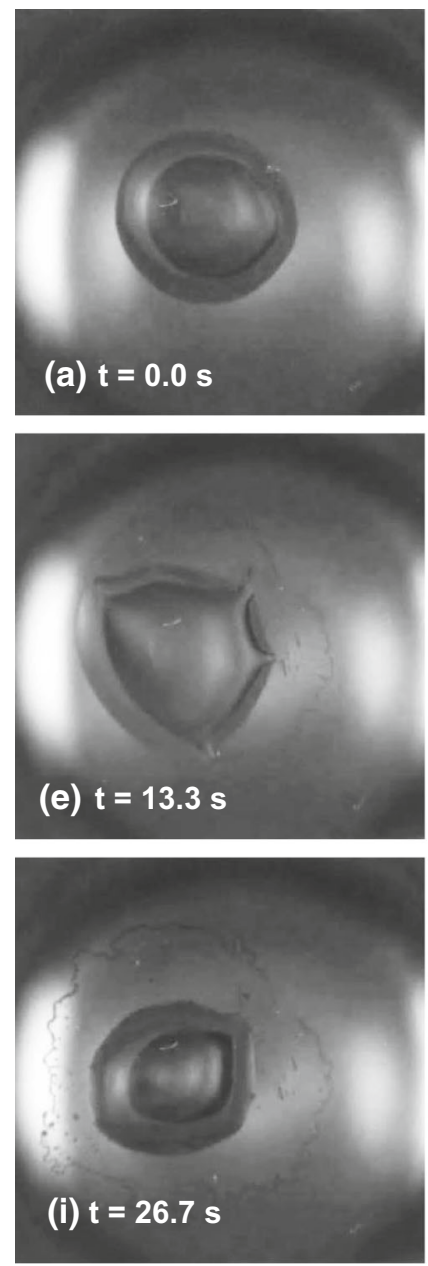
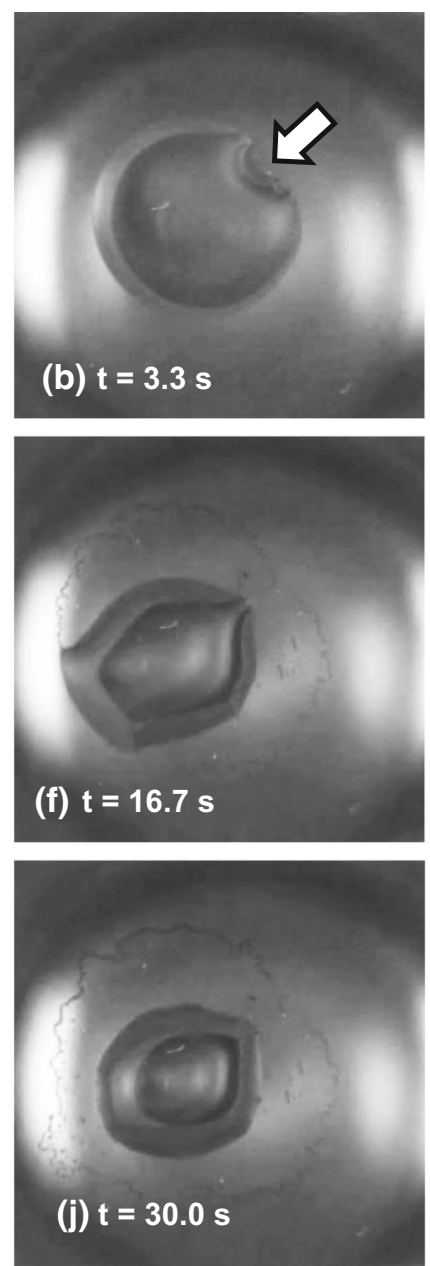
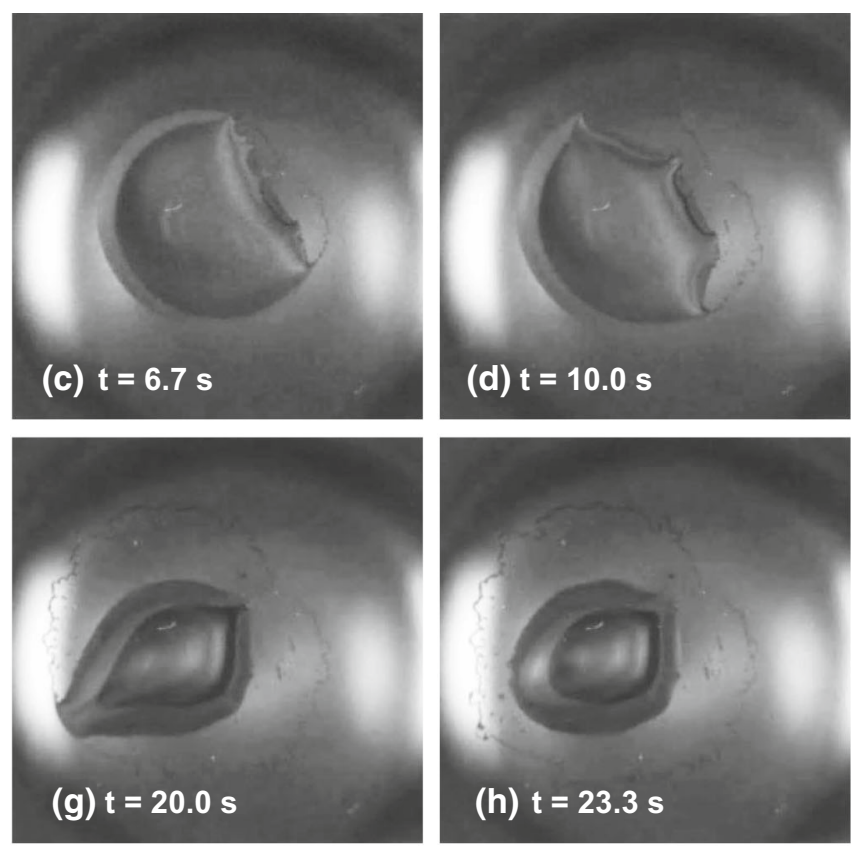

Fig. 4 Video frames of $0.2 \mathrm{wt} \%$ ODA in hexadecane spreading on a silicon surface. No further movement was observed after $30 \mathrm{~s}$

Figs. 4 and $5 a, b$, is an artefact caused by the reflection of the light source in the curved drop, rather than being evidence of a precursor film.

It is interesting that the different behaviours shown in Figs. 4 and 5 are caused by only minor differences in additive concentration. This is because spreading behaviour depends on the balance between the rate of diffusion-driven expansion of solvent on the bare $\mathrm{Si}$ and the rate of additive film formation. It follows from this that adjusting additive concentration can be used to promote a rapid reduction in drop radius thereby confining the lubricant to a specific portion of wafer, which is one of the aims of this study.

Type 4 spreading in which the drop remained motionless on the Si wafer occurred only for hexadecane with $3 \mathrm{wt} \%$ MAC. It was initially speculated that that the MAC may prevent spreading due to the higher viscosity of the MAC/ hexadecane blend compared to neat hexadecane. To test this, a series of spreading tests were performed using different concentrations of squalane in hexadecane, with viscosities close to that of the MAC/hexadecane blend. The results in Fig. 6 show that, as expected, final drop area reduces with increasing viscosity. However, it is also evident that the lack of spreading of MAC blend cannot be attributed solely to its higher viscosity. An alternative hypothesis was that the MAC additive prevented spreading by increasing the surface tension of the blend; however, this possibility was discarded since the measured surface tensions of all the blends were effectively identical (Sect. 2.2). It is therefore suggested that the MAC rapidly forms a film on the Si surface, whose critical surface tension is significantly lower than the blend. It not obvious why the addition of $3 \mathrm{wt} \%$ of a multiply alkylated pentane to hexadecane should form such a film, since this additive contains only carbon and hydrogen atoms and is therefore not expected to be surface active. However, previous research on $\mathrm{Si}$ surfaces, hydrodynamically lubricated by hexadecane-MAC blends, has also shown anomalous behaviour suggesting MACs may indeed be surface active when blended with hexadecane [9]. Furthermore, the research by 

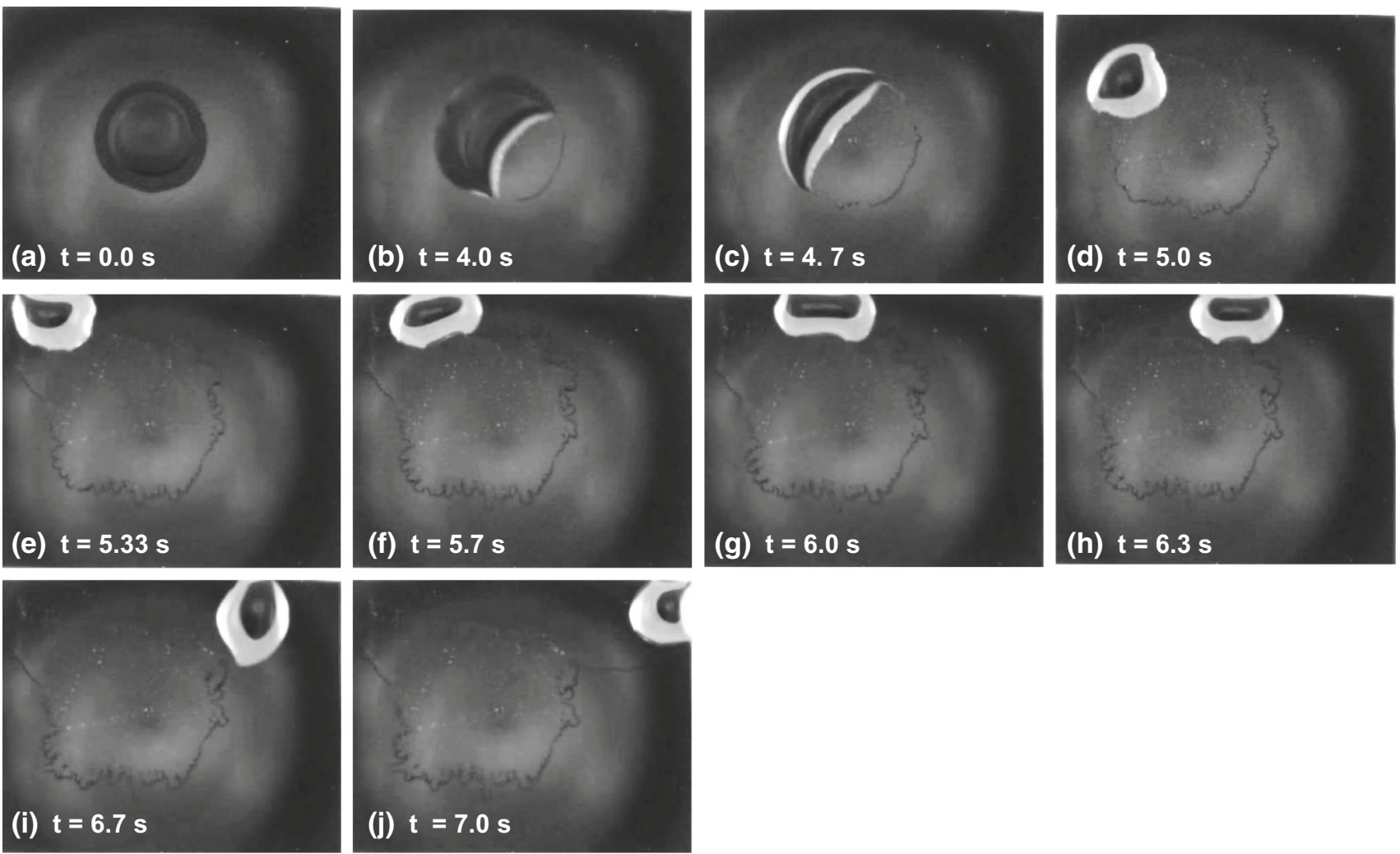

Fig. 5 Video frames of $1 \mathrm{wt} \%$ DDA in hexadecane spreading on a silicon surface. No further movement was observed after $7 \mathrm{~s}$

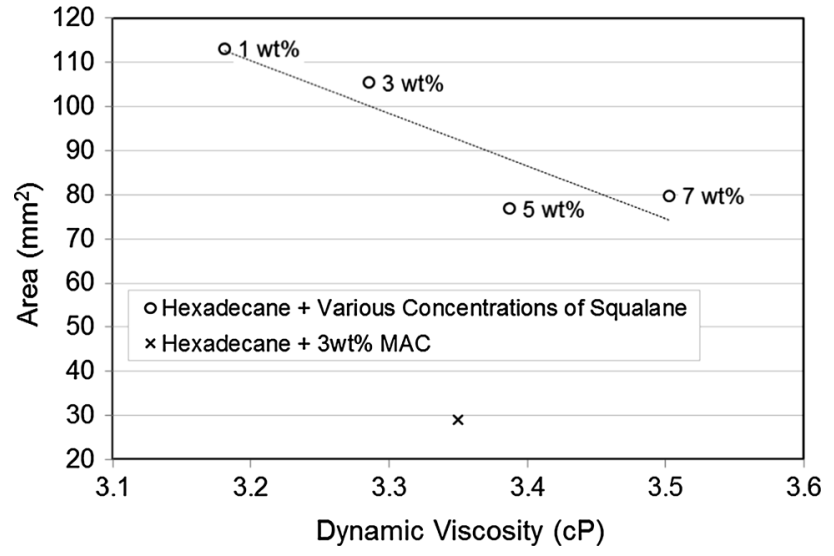

Fig. 6 Plot of steady-state area versus dynamic viscosity for various concentrations of squalane and $3 \mathrm{wt} \%$ MAC in hexadecane

Mate showed that the PFPE lubricant ZDMT—a heavily branched molecule and therefore similar to a MACshowed almost zero spreading [44]. This mode of spreading is most advantageous in terms of lubricant containment.

Figure 7 summarises the spreading and/or retracting behaviour of various hexadecane blends. Here, the effect of the additives in causing the drop to retract is evident, especially in the cases of 0.1 and $0.5 \mathrm{wt} \%$ DDA and $1 \mathrm{wt} \%$ DDA. It is interesting to note that the time until retraction decreases with increasing additive concentration (presumably due to

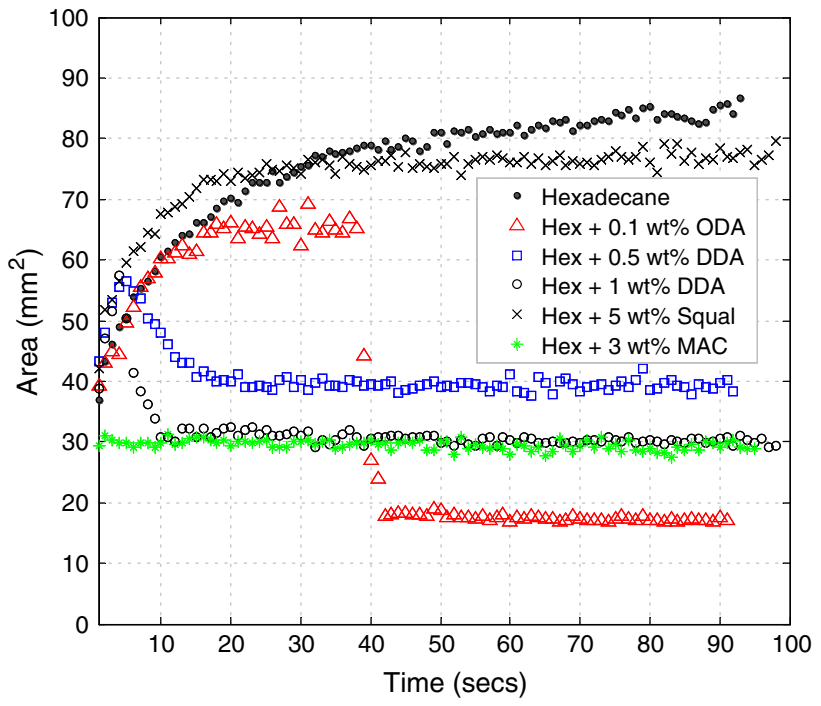

Fig. 7 Plot of drop area versus time for various blends of additives in hexadecane

the increasing rate of film formation). It can also be seen that the film from the $0.1 \mathrm{wt} \%$ blend retracts extremely rapidly (this may be because the drop, having initially expanded to a large diameter, is thin and can flow rapidly over the surface).

All blends, except for the $3 \mathrm{wt} \%$ MAC (which shows no spreading), have approximately the same area upon 
application, with varying times to retraction. The smaller surface area shown after retraction of the ODA blend compared to the DDA blend is predominantly caused by the difference in chain length, which affects the extent of oleophobicity as explained originally by Zisman et al. [17] (note: oleophobicity increases with chain length, since (a) monolayers become more closely packed and (b) the molecules that make up the film become less soluble as chain length increases). The difference in final area between 0.5 and $1 \%$ DDA, on the other hand, is due to the differing rates of film formation.

Lower concentrations such as 0.05 and $0.01 \mathrm{wt} \%$ ODA and DDA are not displayed in Fig. 7 since they did not affect spreading behaviour compared to neat hexadecane. This is consistent with work by Bartell and Ruch [45] who found a marked reduction in contact angle for the low ODA concentration blends that were only sufficient to produce a $50 \%$ complete monolayer.

Figure 8 shows the same data as Fig. 7, except that area, $A$, has been converted to radius, $R,\left(R=(A / \pi)^{0.5}\right)$ and only data points before retraction are shown. Here, the approximately linear nature of the plots shows that the area, and therefore radius of the spreading drop is proportional to the time, $t$, raised to some power, $v$, i.e. $R \sim t^{v}$. This relationship was fitted to the data in Fig. 8 and showed that the mean value of the exponent, $v$, was 0.11 with a standard deviation of 0.012 (for all lubricants excluding the MAC blend). These values are in close agreement with measurements performed by Mate [44], who characterised spreading of PFPE lubricants on carbon-overcoated surfaces and suggested that in the initial stages, spreading is a pressure- rather than a diffusion-driven process. The exception to this behaviour is the hexadecane-MAC blend, which shows zero spreading from the outset, i.e. the droplet

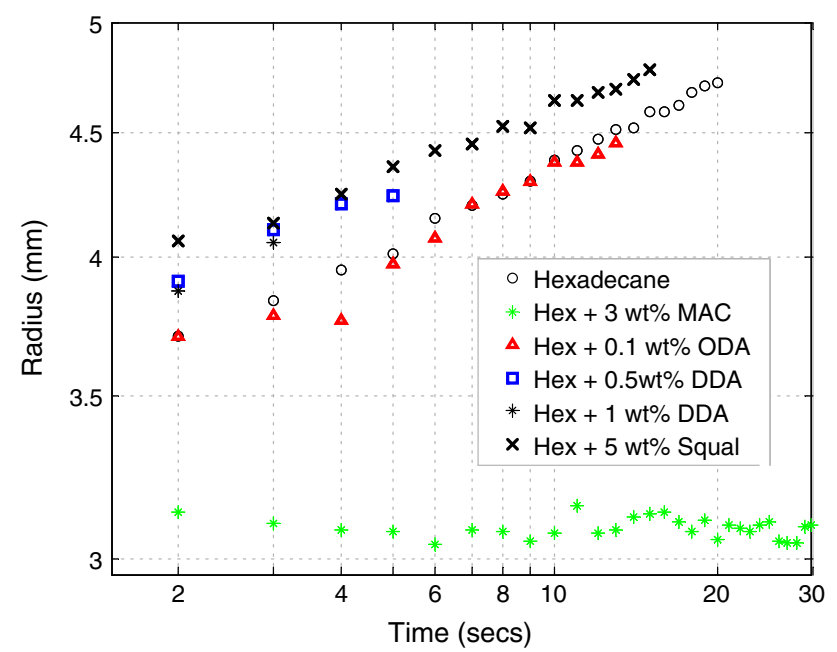

Fig. 8 Log-log plot of drop radius versus time for various blends of additives in hexadecane remains stationary, indicating that the value of the spreading power, $v$, is very close to zero. This suggests that the MAC film forms very rapidly.

This work has focussed on the initial spreading of a drop, immediately after it is placed on a surface. If instead, long-time spreading behaviour were of interest, a curve fitting approach similar to that of [46] would be more appropriate. In this case, an equation of the form $A=A_{0}+C_{1}\left(1-\exp \left(-C_{2} t\right)\right)$ could be fitted to the data in Fig. 7, where $A_{0}$ is the initial drop area, $C_{1}$ is the area growth after a long time and $C_{2}$ is the growth rate coefficient depending on liquid and surface properties.

\subsection{Contact Angle Measurements of Selectively Modified Surfaces}

The contact angle measurements for a selectively modified silicon surface are summarised and compared to an untreated wafer in Table 1 and Figs. 9 and 10. Here, two liquids were used: water and hexadecane. These results show that OTS and plasma treatment are effective in modifying the surface energy of different regions on a single silicon wafer. For instance, the OTS plasma-exposed region has the same surface energy as the treated wafer.

The effect of modification was further tested by gradually increasing the volume of liquid in the circular hydrophilic region. When this region was filled with liquid,

Table 1 Contact angle measurements of modified Si wafer surfaces

\begin{tabular}{lll}
\hline Surface & $\begin{array}{l}\text { Water } \\
\text { contact } \\
\text { angle }\left({ }^{\circ}\right)\end{array}$ & $\begin{array}{l}\text { Hexadecane } \\
\text { contact angle } \\
\left({ }^{\circ}\right)\end{array}$ \\
\hline Plasma cleaned Si & $\sim 0$ & $\sim 0$ \\
Si-OTS (without further treatment) & 106 & 41 \\
Si-OTS after plasma treatment & $\sim 0$ & $\sim 0$ \\
$\begin{array}{l}\text { Unprotected area of Si-OTS } \\
\text { after plasma treatment }\end{array}$ & $\sim 0$ & $\sim 0$ \\
$\begin{array}{l}\text { Protected area of Si-OTS after } \\
\text { plasma treatment }\end{array}$ & 105 & 39 \\
\hline
\end{tabular}
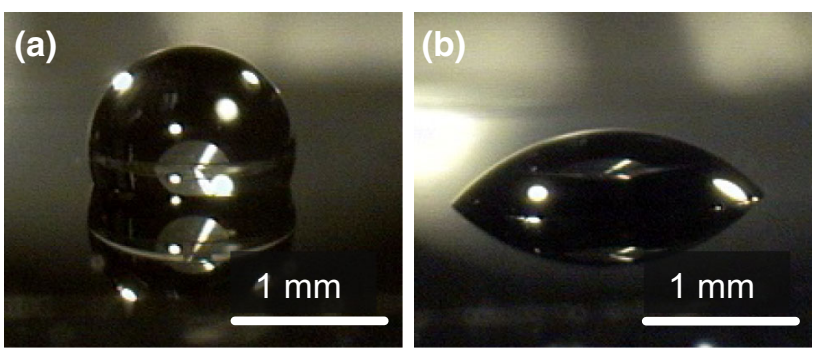

Fig. 9 Photograph of $1 \mu \mathrm{l}$ water droplets on a OTS coated silicon, and $\mathbf{b}$ on the exposed 2-mm-diameter disc section of the selectively modified silicon surface 

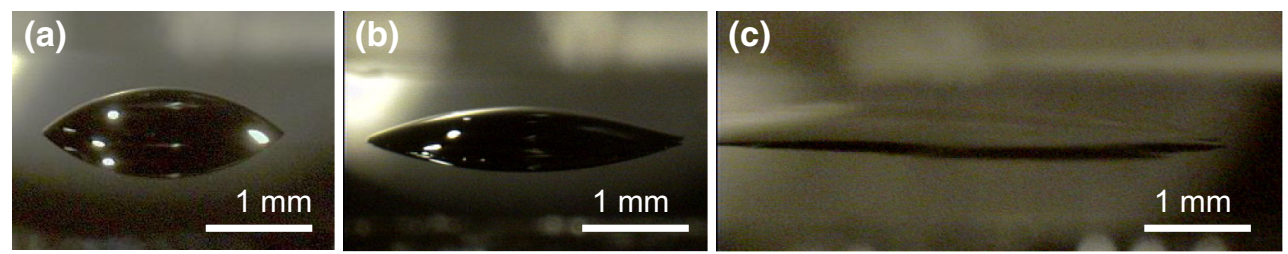

Fig. 10 Photograph of $1 \mu$ l hexadecane drop on a OTS-coated silicon, $\mathbf{b}$ in the exposed 2-mm-diameter disc section of the selectively modified wafer and $\mathbf{c}$ on bare silicon

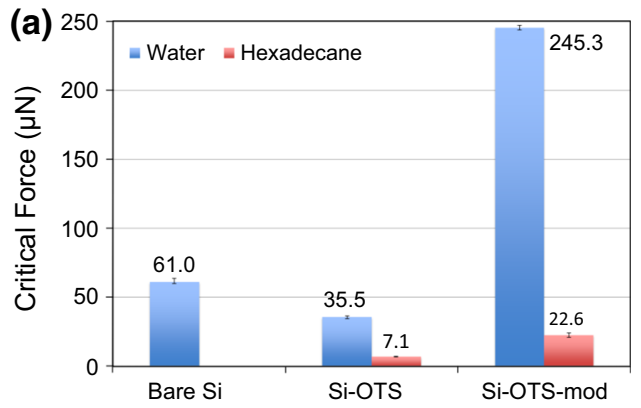

Fig. 11 Critical forces from spin tests, with initial radii of a $20 \mathrm{~mm}$ and b $40 \mathrm{~mm}$, on cleaned bare $\mathrm{Si}$, Si coated with an OTS SAM (Si-OTS), and $\mathrm{Si}$ with selective OTS modification after coating

there was an evident resistance to overflowing into the hydrophobic region. This could be demonstrated by the droplet remaining attached while the wafer was upturned.

\subsection{Spin Tests on Selectively Modified Surfaces}

Summaries of the spin test results are shown in Fig. 11, for radial distances of 20 and $40 \mathrm{~mm}$. These show that selective modification of the surface is highly effective in increasing the force required to remove the lubricant (water or hexadecane) from its initial position on the wafer. The effects are seen more clearly with water than hexadecane, though both liquids show the same trends. The similarity in results between the two sets of results (Fig. 11a, b) highlights the repeatability of the method.

These results are consistent with Eq. 4. Firstly, the step change in surface energy at the drop perimeter (see values in Table 1) means that less energy is lost in forming an solid/liquid interface at the front of the drop compared with the energy expended separating the liquid from the surface at the rear of the drop (i.e. $\gamma_{\mathrm{SL}}^{1}$ is decreased compared to $\gamma_{\mathrm{SL}}^{2}$ leading to a higher $F$ in Eq. 4).

The observed difference in critical force between water and hexadecane also agrees with theory and occurs for two reasons. First, more energy is required to separate the water-Si interface than the hexadecane-Si interface (i.e. $\gamma_{\mathrm{SL}}^{2}$ is higher for water than for hexadecane leading to a higher $F$ in Eq. 4). Second, as demonstrated by the contact

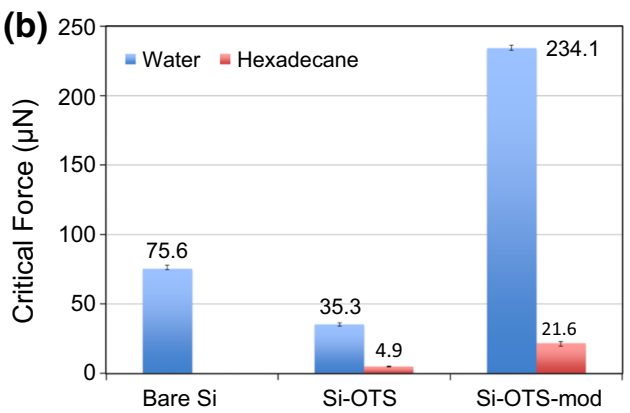

(Si-OTSmod). Note hexadecane spread readily on bare cleaned silicon so no value could be obtained

angles in Table 1, less energy is lost by forming a waterOTS interface than a hexadecane-OTS (i.e. $\gamma_{\mathrm{SL}}^{1}$ is lower for water than for hexadecane leading to a higher $F$ in Eq. 4).

These results confirm that it is the difference in surface energy between adjacent regions which controls how effectively the liquid is contained. Therefore, it should be possible to hold hexadecane drops in place effectively by combining this selective plasma exposure with preparations that produce more oleophobic surfaces [47-50].

The selective modification can also be used to prevent lubricant from entering areas in which flooding would negatively affect functionality of MEMS devices (e.g. electrical pads, comb drives), while keeping the required surface or surfaces lubricated. Another use of such modified surfaces would be to hold a reservoir of liquid at a location separate from the contact, to enable continual replenishment of lubricant via a channel as it is depleted.

\section{Conclusions}

In this work, two methods of confining liquids on silicon surfaces were developed in order to provide a means of supplying lubricant to sliding contacts in MEMS devices.

First, an approach pioneered by Zisman was implemented, in which low concentrations of amphiphilic amine additives were added to hexadecane in order to promote autophobic behaviour. Spreading tests showed that this is 
an effective way of causing drops to retract and reduce in surface area. However, careful tuning of concentration and chain length is required so as not to cause reactive spreading-the situation in which the drop spreads violently driven by a wetting gradient resulting from a partially formed surface film. Factors which control this dynamic behaviour are the speed of formation of the surface film and the degree of wetting that follows. It is also interesting to note that the MAC additive completely prevented the hexadecane drop from spreading (no expansion or retraction was observed once a drop of this solution had been placed on the wafer surface). Further tests demonstrated that this strong effect of the MAC on contact angle was caused neither by an increase in surface tension nor an increase in viscosity. This therefore provides further evidence that the MAC, despite having no functional groups (being simply a blend of di- and tri-(2-octyldodecyl)-cyclopentane), must form some kind of surface film on silicon very rapidly.

Autophobic pinning is effective in limiting the spreading of liquids but does not help liquid drops resist external forces. To overcome this, a method of selective surface modification, previously applied to thiols on gold for micro-contact printing, was successfully adapted to work on $\mathrm{Si}$ surfaces. This approach was highly effective in anchoring hexadecane and water droplets onto the wafer surface. An additional advantage of this method is that it can potentially be used to fabricate lubricant supply channels on the surface of MEMS components. Furthermore, the method of preparation [(1) apply SAMS, (2) mask with PDMS and (3) expose to oxygen plasma] is amenable to batch production and could be incorporated into a MEMS fabrication process.

Both the methods described have the benefit of reducing drop surface area and hence limiting evaporation. This is particularly useful, since liquid MEMS lubricants are necessarily low in viscosity and so tend to have low $\mathrm{MWt}$ and high vapour pressure.

The promise shown by these lubricant containment methods means they may be usefully incorporated into the design of future MEMS bearings, which is the focus of ongoing work.

Acknowledgments This work was partially supported by the Engineering and Physical Sciences Research Council (Grant Number: EP/L001624/1).

Open Access This article is distributed under the terms of the Creative Commons Attribution 4.0 International License (http:// creativecommons.org/licenses/by/4.0/), which permits unrestricted use, distribution, and reproduction in any medium, provided you give appropriate credit to the original author(s) and the source, provide a link to the Creative Commons license, and indicate if changes were made.

\section{References}

1. Yole Development: Status of the MEMS industry 2013 report (2013)

2. Spearing, S.M.: Materials issues in microelectroctromechanical systems (MEMS). Acta Mater. 48, 179-196 (2000)

3. Maboudian, R., Ashurst, W.R., Carraro, C.: Self-assembled monolayers as anti-stiction coatings for MEMS: characteristics and recent developments. Sens. Actuators 82, 219-223 (2000)

4. Smallwood, S.A., Eapen, K.C., Patton, S.T., Zabinski, J.S.: Performance results for MEMS coated with a conformal DLC. Wear 260, 1179-1189 (2006)

5. Asay, D.B., Dugger, M.T., Kim, S.H.: In situ vapor-phase lubrication of MEMS. Tribol. Lett. 29(1), 67-74 (2008)

6. Ku, I.S.Y., Reddyhoff, T., Holmes, A.S., Spikes, H.A.: Wear of silicon surfaces in MEMS. Wear 271, 1050-1058 (2011)

7. Deng, K., Ramanathan, G.P., Mehregany, M.: Micromotor dynamics in lubricating fluids. J. Micromech. Microeng. 4, 266-269 (1994)

8. Ku, I.S.Y., Reddyhoff, T., Choo, J.H., Holmes, A.S., Spikes, H.A.: Lubrication of MEMS devices using liquids of different viscosities. ASME J. Tribol. 134, 0742-4787 (2010)

9. Leong, J.Y., Reddyhoff, T., Sinha, S.K., Holmes, A.S., Spikes, H.A.: Hydrodynamic friction reduction in a MAC-hexadecane lubricated MEMS contact. Tribol. Lett. 49, 217-225 (2012)

10. Reddyhoff, T., Ku, I.S.Y., Holmes, A.S., Spikes, H.A.: Friction modifier behaviour in lubricated MEMS devices. Tribol. Lett. 41, 239-247 (2010)

11. Rosen, M.J.: Surfactants and Interfacial Phenomena, 3rd edn. Wiley, New York (1989)

12. Fowkes, F.M.: Contact Angle, Wettability, and Adhesion. American Chemical Society, Washington (1964)

13. Bonn, D., Eggers, J., Meunier, J., Indenkeu, J., Rolley, E.: Wetting and spreading. Rev. Mod. Phys. 81(2), 739-805 (2009)

14. Wisner, G., Brevet, F.G.: Improvement in the lubrication of mechanical components, Cl.3 No 621.077, 11.06 (1925)

15. du Parquet, J.: French contribution to the study of lubrication, oiliness, molecular influences, application to watch lubrication. In: Dowson, D. (ed.) Lubrication at the Frontier: the Role of the Interface and Surface Layers in the Thin Film and Boundary Regime. Institut des Sciences Appliquées de Lyon, p. 621 (1998)

16. Bulkley, R., Snyder, G.H.S.: Spreading of liquids and solid surface. The anomalous behaviour of fatty oils and fatty acids with experiments leading to a tentative explanation. J. Am. Chem. Soc. 55, 194-208 (1933)

17. Bigelow, W.C., Pickett, D.L., Zisman, W.A.: Oleophobic monolayers: I. Films adsorbed from solution in non-polar liquids. J. Colloid Sci. 1(6), 513-538 (1946)

18. Hare, E.F., Zisman, W.A.: Autophobic liquids and the properties of their adsorbed films. J. Phys. Chem. 59(4), 335-340 (1954)

19. Timmons, C.O., Zisman, W.A.: A study of autophobic liquids on platinum by the contact potential method. J. Phys. Chem. 68(6), 1336-1342 (1964)

20. Bernett, M.K., Zisman, W.A.: Prevention of liquid spreading or creeping. In: Gould, R.F. (ed.) Contact Angle, Wettability, and Adhesion. American Chemical Society, Washington (1964)

21. Biebuyck, H.A., Whitesides, G.M.: Autophobic pinning of alkanethiols on gold. Langmuir 10, 4581-4587 (1994)

22. Murray, R.J., Boettinger, B.T., McFadden, W.J., Braun, G.B.: Theory of reactive spreading of a thin drop. Phys. Fluids 7(8), 1797-1810 (1995)

23. Quéré, D., Azzopardi, M.-J., Delattre, L.: Drops at rest on a tilted plane. Langmuir 14, 2213-2216 (1998)

24. Berejnov, V.V.J.: Self pinning protein-laden drops. Colloid Interface Sci 322, 246-253 (2008) 
25. Yu, H., Zhou, G., Sinha, S.K., Leong, J.Y., Chau, F.S.: Characterization and reduction of MEMS sidewall friction using novel microtribometer and localized lubrication method. J. Microelectromech. Syst. 20(4), 991-1000 (2011)

26. Sinha, S.K. et al.: Method of applying a lubricant to a micromechanical device. US Patent US 20130071629, Mar 21, 2013

27. Rymuza, Z.: Tribology of Miniature Systems. Elsevier Science, Oxford (1989)

28. Mate, C.M.: Tribology on the Small Scale: a Bottom up Approach to Friction, Lubrication, and Wear. Oxford University Press, Oxford (2008)

29. Brochard-Wyart, F., Quéré, D., De Gennes, P.: Capillarity and Wetting Phenomena: Drops, Bubbles, Pearls. Springer Science \& Business Media, New York (2004)

30. Zisman, W.A.: Relation of the equilibrium contact angle to liquid and solid constitution. Adv. Chem. Ser. 43, 1-51 (1964)

31. Wolfram, E., Faust, R.: Liquid Drops on a Tilted Plate, Contact Angle Hysteresis and the Young Contact Angle, pp. 213-222. Academic Press, New York (1978)

32. Eral, H.B., Oh, J.M.: Contact angle hysteresis: a review of fundamentals and applications. Colloid Polym. Sci. 291(2), 247-260 (2013)

33. Lichao, G., McCarthy, T.J.: Contact angle hysteresis explained. Langmuir 22(14), 6234-6237 (2006)

34. Guo, L., Wong, P.L., Guo, F.: Correlation of various wetting parameters with hydrodynamic lubricating effects. Leeds, p. 23.4 (2014)

35. Wang, Y., Yufei, M., Zhu, M., Bai, M.: Wettability and nanotribological property of multiply alkylated cyclopentanes (MACs) on silicon substrates. Trib. Trans. 53(2), 219-223 (2010)

36. Gianino, C.: Measurement of surface tension by the dripping from a needle. Phys. Educ. 41(5), 440-555 (2006)

37. Rena, S., et al.: Friction and wear studies of octadecyltrichlorosilane SAM on silicon. Tribol. Lett. 13(4), 233-239 (2002)

38. Myo, M., Leong, J.Y., Sinha, S.K.: Effects of interfacial energy modifications on the tribology of UHMWPE coated Si. J. Phys. D Appl. Phys. 41(5), 055307 (2008)
39. Myo, M.: Tribology of Polymer Film with Hard Interlayers on Si Surface-Role of Surface Energy on Adhesion and Static Friction, National University of Singapore, $\mathrm{PhD}$ Thesis (2009)

40. Lin, M.H., Chen, C.F., Shiu, H.W., Chen, C.H., Gwo, S., Lin, M.-H., Chen, C.-F., Shiu, H.-W.: Multilength-scale chemical patterning of self-assembled monolayers by spatially controlled plasma exposure: nanometer to centimeter range. J. Am. Chem. Soc. 131(31), 10984-10991 (2009)

41. Cottington, R.L., Murphy, C.M., Singleterry, C.R.: Effect of polar-nonpolar additives on oil spreading on solids, with applications to nonspreading oils. In: Gould, R.F. (ed.) Contact Angle, Wettability, and Adhesion, pp. 341-354. American Chemical Society, Washington (1964)

42. Hunter, J.K., Li, Z., Zhao, H.: Reactive autophobic spreading of drops. J. Comput. Phys. 183, 335-366 (2002)

43. Pesach, D., Marmur, A.: Marangoni effects in the spreading of liquid mixtures on a solid. Langmuir 3(4), 519-524 (1987)

44. Mate, C.M.: Spreading kinetics of lubricant droplets. Tribol. Lett. 55, 385-395 (2013)

45. Bartell, L.S., Ruch, R.J.: The wetting of incomplete monomolecular layers. II Correlation with molecular size and shape. J. Phys. Chem. 63(7), 1045-1049 (1959)

46. Brunner, R., Etsion, I., Talke, F.E.: Long time spreading of a microdroplet on a smooth solid surface. Langmuir 26(3), 1824-1829 (2010)

47. Sagiv, J.: Organized monolayers by adsorption. 1. Formation and structure of oleophobic mixed monolayers on solid surfaces. J. Am. Chem. Soc. 102(1), 92-98 (1980)

48. Liu, Y., Xiu, Y., Hess, D.W., Wong, C.P.: Silicon surface structure-controlled oleophobicity. Langmuir 26(11), 8908-8913 (2010)

49. Juhue, D, Pabon, M., N'Zudie, D.T., Corpart, J.M., Lina, M.-J.: Fluorocopolymers for the hydrophobic and oleophobic treatment of various substrates. Grant 6518380 (2003)

50. Tuteja, A., et al.: Designing superoleophobic surfaces. Science 318(5856), 1618-1622 (2007) 